\title{
PODER, NORMAS SOCIALES Y DESIGUALDAD DE LAS MUJERES EN EL HOGAR
}

\author{
Power, Social norms and Inequality \\ of Women in the Household
}

Arlette Covarrubias Feregrino ${ }^{1}$

Fecha de recepción: 30 de junio de 2016 Fecha de aceptación: 27 de octubre de 2016 


\section{Resumen}

Este artículo propone un marco teórico para analizar el poder de las mujeres en el hogar. Se hace especial énfasis en la visibilidad del poder y en los distintos mecanismos utilizados para mantenerlo, ya que éstos tienen importantes consecuencias en el bienestar de los miembros del hogar, en especial de las mujeres. De esta forma, el artículo se basa en el marco teórico de empoderamiento propuesto por Kabeer (1999) y las tres formas de poder sugeridas por Gaventa (2011), quien retoma las tres dimensiones de poder de Lukes (2005). Debido a que las normas sociales son un factor fundamental del poder de los miembros del hogar, y lo influyen a través de varios canales, se utiliza el marco conceptual propuesto, para analizar las varias formas en que éstas influyen en el poder de los miembros del hogar.

Palabras clave: poder, normas sociales, empoderamiento de las mujeres, capacidades, agencia.

\section{Abstract}

This article proposes a theoretical framework to analyse women's power in the household. Given the important consequences they have on household members welfare, especially that of women, special emphasis is made the visibility of power and the different mechanisms used to maintain it. Thus, this article draws from the framework of empowerment proposed by Kabeer (1999) and the three forms of power suggested by Gaventa (2011), which are based on the three dimensions of power by Lukes (2005). As social norms are a fundamental factor which influences power through several channels, the proposed framework is used to analyse the several ways in which social norms affects household members' power.

Keywords: power, social norms, women's empowerment, capabilities, agency. 


\section{Introducción}

$\mathrm{L}$

a equidad y bienestar de las mujeres tiene un valor intrínseco fundamental e importantes implicaciones para el desarrollo social, político y económico de un país. El empoderamiento, que se refiere la capacidad de una persona de tomar elecciones estratégicas de vida, ha sido promovido por feministas y organizaciones de desarrollo como una estrategia complementaria a la protección social para alcanzar este objetivo. Para que esta estrategia sea exitosa, es fundamental que se analicen a profundidad cuáles son los factores, mecanismos y dinámicas con las cuales las mujeres tienen mayores oportunidades para empoderarse, ya sea en el ámbito individual, del hogar, comunitario, nacional, o a nivel internacional.

Este artículo se propone ahondar en el poder relacional de las parejas en el hogar. Las dinámicas de poder en este ámbito son cruciales para el bienestar de los individuos, ya que determinan aspectos fundamentales de su vida. En primer lugar, influyen en la distribución del ingreso, de bienes y de servicios en el hogar, y con esto la posibilidad de estar bien nutridos, de tener vivienda, de contar con cuidados, etc. En segundo, repercuten en las actividades productivas, reproductivas y de ocio, a las que se dedican los miembros del hogar, y con esto el acceso a recursos sociales, humanos y económicos a los que pueden acceder, y a la satisfacción que trae consigo el involucramiento en estas tareas. Tercero, repercuten en logros básicos no pecuniarios a los que tiene acceso una persona como lo es la movilidad, la afiliación con otras personas, la salud reproductiva, la satisfacción sexual, etc. En México, las mujeres cuentan con menos oportunidades de adquirir estos logros, debido a las relaciones de poder asimétricas que tienen con hombres en el hogar y que están determinadas sustancialmente por las normas sociales de género.

Usando el marco conceptual de Capacidades elaborado por Sen (1985a, 1985b, 1987b, 1990, 1992, 1993, 1999), Kabeer (1999) desarrolló un marco para el análisis del empoderamiento de las mujeres que es ampliamente citado. En él, el empoderamiento depende de los recursos sociales, humanos y económicos que los individuos tienen y de su agencia, que es la habilidad que tiene una persona de definir sus propios objetivos y de actuar en consecuencia. Este marco provee una base elemental para el estudio del empoderamiento de las mujeres, pero no considera, que en el hogar, surgen conflictos de interés entre sus miembros, y su resolución depende de los mecanismos y visibilidad del poder al alcance de ellos. Estas dinámicas, influirán también en el bienestar de las personas, ya que si, por ejemplo, son violentas, influirán en su integridad, en su salud, y en su posibilidad de vivir sin miedo y ansiedad. En cambio, si los miembros tienen posibilidad de negociar y de entablar con otros una reflexión crítica, experimentarán un bienestar emocional.

En este artículo se elabora un marco teórico para el análisis del poder en las relaciones del hogar usando las formas de poder de Gaventa (2011) que están basadas en las tres dimensiones de poder de Lukes (2005), estableciendo también los mecanismos que se pueden utilizar para mantener y ejercer poder. Con este marco, pueden analizarse el efecto de los determinantes del poder de las mujeres y las estrategias para empoderarlas, de una forma más precisa y clara.

Uno de los factores más importantes de poder de las personas son las normas sociales. En varias comunidades estas normas establecen la jerarquía de los esposos en el hogar. Éstas, también son usadas como argumentos para validar posturas. Establecen además cuál debe ser el comportamiento de los miembros del hogar a la hora de negociar. Influyen en que las mujeres tengan un menor acceso a los factores que las empoderan, es decir, a recursos y a su agencia. Por lo tanto, son varias las vías a través de 
las cuales las normas sociales influyen en el poder de las esposas en el hogar. Dada su importancia y con el fin de demostrar la utilidad del marco teórico propuesto, se analiza cómo es que estas normas influyen en las formas, mecanismos de poder, en los recursos y la agencia.

Agarwal (1997), elaboró teóricamente sobre los medios a través de los cuales las normas sociales influyen en la negociación de los miembros del hogar. Precisa que las normas sociales indican los límites sobre lo que puede ser negociado, son un determinante del poder de negociación, afectan el proceso de negociación, y constituyen un factor que es negociado. No obstante, no considera que en el hogar, muchos intereses no serán explícitamente negociados, y que las normas sociales, influirán en los mecanismos usados en las dinámicas de poder.

Este artículo tiene entonces, el objetivo de ahondar teóricamente en las dimensiones y mecanismos para mantener y ejercer el poder en el hogar e indagar cómo las normas sociales influyen en éstas, en primer lugar se ahonda en la definición y conceptualización de poder, y se propone un marco para analizar el poder en el hogar. La tercera sección elabora en la definición y descripción de las normas sociales. Por último, se usa el marco propuesto para relacionar las normas sociales en el poder del hogar.

\section{Poder y empoderamiento de las mujeres en el hogar}

La definición del concepto de poder, ha sido ampliamente debatida. De acuerdo con Lukes (2005), la controversia en torno a la definición de esta palabra se debe a que es polisémica, tiene varios significados que son usados de forma distinta dependiendo del contexto y la aplicación. Existe una disimilitud importante en las conceptualizaciones de poder, que es fundamental mencionar. El concepto de poder puede ser usado como 'poder sobre' alguien o como 'poder expansivo de libertades'. Utilizando la distinción que hace Spinoza (2002), Lukes (2005) indica que esto se debe a que proviene de dos raíces latinas:

Potentia: que significa el poder que tienen las cosas en la naturaleza, incluyendo a las personas, de 'existir y actuar'.

Potestas: es usada en referencia a tener poder sobre otra persona.

Potestas, es entonces un subconjunto de potentia. Cabe resaltar que el término 'empoderamiento' usado por feministas, se basa en la raíz 'potentia', por lo que el proceso de empoderamiento de una persona no necesariamente implica desempoderar a otra, es decir, el poder no es juego de suma-cero donde algunos pierden y otros ganan.

De acuerdo con el concepto de poder 'potentia', Rowlands (1997) clasifica las distintas formas de poder de la siguiente manera:

- Poder sobre: Poder controlador, que puede ser respondido con docilidad, resistencia (que debilita el proceso de victimización), o manipulación.

- Poder para: poder productivo o generativo (a veces incorporando o manifestado como formas de resistencia o manipulación) que crea nuevas posibilidades de acción sin dominación.

- Poder con: Se refiere al efecto de sinergia. El conjunto es mayor que la suma de individuos, especialmente si el grupo resuelve los problemas juntos. 
- Poder desde dentro: Es la fuerza espiritual interior que reside en cada uno de nosotros y nos hace humanos. Es la base de auto aceptación y auto respeto que por su parte respeta y acepta a los otros como iguales.

Se deriva que las relaciones de poder operan a distintos niveles en la sociedad, a nivel individual, familiar, comunitario, nacional, o a nivel internacional (Malhotra et al., 2005). Por lo tanto, los individuos y los grupos de personas buscan empoderarse en cada una de estas esferas, y el poder en alguno de los niveles, influye en el poder que se tiene en los otros.

Como ya se ha mencionado, este artículo se centra en el poder relacional entre esposos (o aquellos que viven en unión libre), es decir, en la habilidad de restringir las opciones de los individuos. En el caso de que las mujeres vivan con la familia de sus esposos, como es común todavía en varias comunidades, la esposa tendrá que negociar decisiones importantes no sólo con él, sino también con los miembros de su familia extendida (Appendini, 2009). No obstante, con el fin de simplificar el análisis, se hará abstracción de las relaciones con el resto de la familia, y se enfocará el análisis en las decisiones entre esposos. Para este efecto, primero se analizará el concepto de poder utilizado por las feministas cuando se refieren al empoderamiento, y se ahondará en la insuficiencia del término para analizar el poder y empoderamiento de las mujeres a nivel relacional en el hogar.

Basado en el marco teórico de capacidades de Sen (1985a, 1985b, 1987b, 1990, 1992, 1993, 1999). Kabeer (1999) desarrolló un marco teórico de empoderamiento, donde la habilidad para ejercer elecciones es considerada en términos de tres dimensiones interrelacionadas; los recursos con los que cuenta un individuo, su agencia y sus logros.

El empoderamiento es conceptualizado como la expansión de las capacidades individuales y colectivas de las personas excluidas y marginalizadas, para que puedan así reclamar efectivamente sus derechos humanos y participar, negociar, influir y responsabilizar a los actores que afectan su acceso a tales derechos. Por ende, implica la expansión de la habilidad de un individuo o grupo de personas para tomar elecciones, en un contexto donde esta habilidad había sido previamente negada (Kabeer, 1999). El empoderamiento debe implicar mayor libertad para todos, mayor equidad, y mayor autodeterminación (Sen, 1999).

Hay dos puntos que deben de recalcarse. En primer lugar, el empoderamiento se refiere a decisiones estratégicas de vida. Esto es, decisiones que son críticas para que las personas puedan vivir la vida que deseen. En segundo lugar, el empoderamiento implica un proceso de cambio. Para que un individuo se empodere, inicialmente tiene que estar desempoderado, de manera tal que las habilidades de hacer decisiones estratégicas hayan sido inicialmente denegadas o limitadas (Kabeer, 1999).

Al hablar de recursos, Kabeer (1999) se refiere no solo a los recursos económicos, sino también a los recursos humanos y sociales que juegan un papel importante en la posibilidad de que una persona pueda tomar una 'elección'. Éstos son adquiridos a través de una multiplicidad de relaciones sociales que se llevan a cabo en varias instituciones, tales como la familia, el mercado, la comunidad, etc. Por otra parte, la 'agencia' es la habilidad que tiene una persona de definir sus propios objetivos y de actuar en consecuencia. La agencia se refiere a más que una acción aislada, también, incorpora la noción de significado, motivación y propósito con el que los individuos actúan, es decir, su poder desde dentro y éste puede ser ejercido por individuos y colectividades. 
Los recursos y la agencia de una persona determinan sus Capacidades, esto es, el potencial que tiene una persona de vivir la vida que quiere, de alcanzar múltiples formas de funcionalidades, es decir, de "ser y hacer." Los logros se refieren a estas múltiples formas de 'ser y hacer' que una persona ha alcanzado. Por ejemplo, si una persona ha alcanzado estar nutrida, saludable, educada, etc. En suma, el empoderamiento o capacidad de una persona de tomar decisiones, implica que ésta tenga alternativas de donde escoger, que a su vez depende de la agencia y los recursos con que cuenta una persona. La figura 1, muestra las tres dimensiones interrelacionadas de la habilidad de ejercer elecciones; los recursos, la agencia y los logros de los individuos.

\section{Figura 1. Proceso de empoderamiento de los individuos}

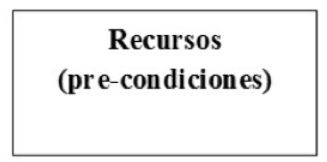

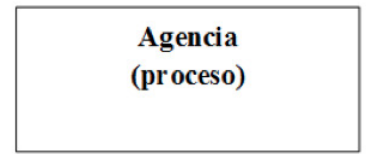

Fuente (Kabeer, 1999: 437)

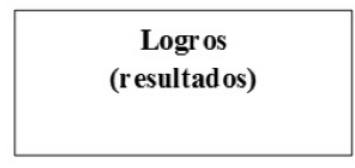

nivel relacional, es posible tener un poder para o poder productivo, en el que ambas personas, por ejemplo, suman su esfuerzo para resistir la dominación de una persona externa a la pareja. También pueden tener juntos un efecto sinergia, una agencia conjunta, en donde ambos eligen y resuelven los problemas de forma colectiva. En este caso, se manifiesta la agencia a la que hace referencia Kabeer (1999). Contrariamente, pueden surgir dinámicas de poder de uno sobre otro, es decir que un miembro de la familia limite las opciones y elecciones que están a disposición del otro.

Esto ya lo había observado Sen (1987a), quien indicó que, en el hogar, por una parte, las capacidades que un individuo adquiere dependen de los otros miembros del hogar. Por otro lado, hay también conflictos de necesidades, intereses y preferencias. "Los miembros del hogar enfrentan dos tipos de problemas simultáneamente, uno que involucra la cooperación, (que añade a las disponibilidades) y otro el conflicto (la división de las disponibilidades entre los miembros del hogar) (Sen 1987a:12)." Por lo tanto, al hacer comparaciones interpersonales es necesario reconocer que las capacidades en muchas ocasiones tienen una dimensión interdependiente, especialmente cuando se habla del hogar (Iversen, 2003).

Los logros de los miembros del hogar dependerán entonces del poder que tengan sobre el otro cuando surjan los conflictos en este ámbito y el marco conceptual de empoderamiento de Kabeer (1999), no considera esta potencial discrepancia. Si se tiene como objetivo empoderar a las mujeres en el hogar, es importante que se analicen a profundidad, las formas, mecanismos y factores que influyen en las dinámicas del poder entre ellas y sus parejas, incluyendo el conflicto. Este artículo tiene como propósito desarrollar un marco explicativo a partir de la propuesta de Kabeer (1999), que incorpore los elementos anteriormente mencionados, adicionando el marco conceptual de Lukes (2005) y Gaventa (2011).

En el marco que delinea Lukes (2005), se distingue conceptualmente tres dimensiones del poder. Gaventa (2011) las retoma, pero las clasifica como tres formas de poder, cada una diferenciada por su nivel de visibilidad. Cabe aclarar que ambos autores formulan su clasificación considerando al poder en el ámbito político, y en el presente artículo, se adecúa para construir un marco que permita analizar el poder relacional. De esta forma, las formas de poder se distinguen de la siguiente manera: 
- La forma visible: involucra decisiones controversiales sobre las cuales hay un conflicto explícito y observable. El conflicto remite a preferencias presumiblemente conscientes, exhibidas en acciones y pueden descubrirse observando los actos de los individuos. Al ver quien participa, cuáles son los intereses que se debaten, que voces están presentes, pero tienen poca influencia, quien pierde y quien gana, se puede dilucidar quien tiene poder. En esta forma de poder, los actores son conocedores de sus agravios y son capaces de articularlos, es decir, tienen la agencia necesaria, además de los recursos, para entrar en el proceso de deliberación. En el caso relacional de pareja, en esta forma de poder, ambas personas entran en el proceso de deliberación, manifiestan sus inquietudes y deseos, y se presume que aquel que tiene poder es el que consigue que se logre su interés. En esta forma de poder, se pone escasa atención a aquellos que no tienen voz y que no entran en el proceso de deliberación.

- El poder oculto. Este tipo de poder se refiere a la creación de barreras que previenen la participación en la toma de elecciones. Involucra mecanismos en las que una persona previene a otra de sacar a relucir un conflicto. También incluye acciones en las que las personas resisten la dominación de forma menos pública. En el caso de las parejas, este tipo de poder se manifiesta cuando alguno de los integrantes, a menudo la mujer, no expresa ni negocia sus intereses explícitamente. En el caso del empoderamiento de las mujeres, resulta fundamental distinguir entre este tipo de poder y el poder visible, ya que implica que el individuo carece de la agencia suficiente o la esperanza de posicionar sus intereses, usando mecanismos públicos y explícitos. Las estrategias que abordan este tipo de poder se enfocan en potenciar la voz de las personas y su capacidad de expresar y manifestar sus necesidades.

- El poder invisible: Aunque es menos visible, el poder oculto, asume que las personas están conscientes y son capaces de articular sus agravios y deseos. Sin embargo, el poder invisible involucra formas en las cuales el conocimiento de los derechos e intereses son escondidos a través de ideologías, formas de valor y comportamiento que están determinadas por patrones culturales, sociales y políticas de instituciones. Tiene que ver con la "internalización” de las personas en formas que afecta su conocimiento y consciencia de asuntos y conflictos potenciales. Sen (1987a) indica que una persona puede percibir que su bienestar tiene menor valor que el de otros. En ciertas sociedades tradicionales, las mujeres pueden internalizar que es más importante el bienestar de su familia que la suya. Esta forma de poder es fundamental cuando se analiza el poder de las mujeres, no sólo porque afecta sus preferencias percibidas, pero también debido a que las mujeres internalizan a tal grado sus roles de esposa y madres, que pueden no cuestionar las mismas. Más adelante se discutirá este aspecto a mayor profundidad.

El poder invisible, ha sido debatido, ya que se puede confundir el significado de este concepto, al implicar que los individuos pueden tener una "conciencia falsa." Este concepto en términos marxistas, se refiere a una persona que no sabe cuáles son sus verdaderos intereses, pero sí hay actores 
externos y privilegiados que sí pueden saber cuáles son los intereses de ese individuo (Lukes 2005). Esta posición es de condescendencia con la persona. Sin embargo, como Kabeer (1999: 441) indica, no se trata de indicar si hay una conciencia verdadera o falsa, sino de "cómo las personas perciben sus necesidades e intereses dependiendo de su historia individual y su realidad cotidiana, por sus contextos materiales y sociales de sus experiencias y por la perspectiva ventajosa de reflexividad que esta provee." Hay necesidades e intereses que no son evidentes debido a que están inscritas en reglas, normas y costumbres sociales que están dadas por sentadas.

Debido a que las estrategias para empoderar a los individuos difieren dependiendo de la visibilidad del poder, es fundamental distinguir entre estas tres formas de poder no sólo en el escenario político sino también en las relaciones. Debe indicarse que, aunque es importante separar conceptualmente estas tres formas de poder, en la práctica estas están interrelacionadas y varían en el tiempo (Gaventa, 2011).

Además de distinguir las formas de poder, también es útil, especialmente cuando se analiza el poder a nivel relacional, distinguir entre los mecanismos que pueden ser utilizados para mantener el poder y para resistirlo. Esto es, debido a que algunos de los mecanismos de poder, pueden ser perniciosos, limitando así el bienestar de los individuos. Para efectos del análisis, los mecanismos de poder utilizados están basados en modificaciones de las categorizaciones de Lukes (2005) y Scott (2001). En las formas de poder visible y oculto, los mecanismos de poder son, la fuerza, la coerción, la influencia, la autoridad, y la manipulación.

Coerción: El establecimiento de una estructura de poder a través de la coerción, reside en la amenaza del uso de la fuerza (el uso de sanciones físicamente o emocionalmente restrictivas para prevenir las acciones de los subalternos) o de una privación seria por parte de la persona que ejerce poder sobre otra, y en una creencia por parte del subalterno, que éste tiene tanto la capacidad como la disposición de usarla. La amenaza de usarla cambia la estructura de costos y beneficios asociados a cursos específicos de acción (Scott, 2001).

Manipulación: tiene que ver con el uso del engaño y la mentira para mantener el poder. Como indicaría Lukes (2005: 22) "la manipulación es un aspecto o sub concepto de la fuerza, donde la conformidad reside en la ausencia de reconocimiento por parte del que actúa en aquiescencia, de la exacta naturaleza de la demanda que se le hace".

Autoridad: La conformidad de la autoridad esta sostenida por la idea de que se tiene el derecho de impartir ordenes, y una correspondiente obligación de obedecer. Existe cuando una o varias personas tácita o explícitamente permiten que alguien más tome decisiones por ellos en algunas acciones. Un individuo tiene la disposición a conformarse debido a la legitimidad de la fuente del mando y no debido a una evaluación del contenido de la acción o situación a la que se conforma. El poder es aceptado ya que se considera lo correcto, justificado o válido, y su legitimidad fluye de la internalización de significados culturales (Scott, 2001).

Influencia: cuando la persuasión opera a través de símbolos cognitivos, las ideas y representaciones que llevan a que las personas definan situaciones en una forma específica, toma la forma de significación. En este caso, los individuos son atraídos e influenciados por el marco de referencia interpretativo del principal (Scott, 2001).

Tanto la influencia como la autoridad, operan a través del ofrecimiento y la aceptación de razones para actuar de una forma o de otra. Depende de símbolos cognitivos socialmente estructurados que están basados 
en raciocinios sobre la acción de otros, y que son considerados intrínsecamente apropiados y plausibles. El curso de acción es calificado como moral o emocionalmente apropiado y depende de argumentos, solicitudes y razones que hacen que el individuo considere más apropiado actuar de una forma que de otra (Scott, 2001).

De los mecanismos de poder se evidencia, que hay algunos más perniciosos que otros. La coerción a través de la violencia física, es más perjudicial que el uso de la persuasión a través de razonamientos y argumentos. Resulta entonces, fundamental conocer los mecanismos que utilizan unas personas para ejercer poder sobre otros al interior de los hogares.

Por otra parte, una sociedad, persona o institución puede ejercer poder invisible sobre otras, manipulando o transformando sus deseos a través de los medios de comunicación e información, así como de los procesos de socialización. Las estrategias para contrarrestar el poder invisible, involucran la creación de consciencia, la educación adulta, el uso de los medios de comunicación y campañas para desafiar los estereotipos, normas sociales y discursos, así como también cambios en los métodos de enseñanza (Gaventa, 2011). Debido a que estos patrones culturales de comportamiento no están establecidos en un momento dado por los miembros del hogar, no se puede hablar del poder de un miembro sobre otro. No obstante, en virtud de que la libertad de uno de los integrantes del hogar es restringida, resulta evidente el ejercicio del poder.

El poder invisible intersecta con el concepto de normas y roles sociales, ya que estos influencian los deseos y el proceso de toma de decisión de los individuos. No obstante, hacer una distinción explicita entre el poder y las normas sociales y elaborar teórica y empíricamente su interacción, es de gran utilidad en el momento de establecer y explicar los factores que influyen en el empoderamiento de las personas y en determinar políticas sociales a combatirlas. Las normas sociales, por ejemplo influyen también en las manifestaciones de poder en la primera y segunda dimensión. En una discusión entre esposos, donde el poder es visible, estos harán uso de los roles sociales que cada uno debe cumplir para validar sus argumentos: por ejemplo, el varón puede argumentar que su mujer no debe salir a vender mercancía a un pueblo lejano ya que es su obligación de ama de casa hacer la comida.

En la figura 2 se resume la conceptualización de poder relacional. El poder relacional de los miembros del hogar dependerá tanto de su agencia como de los recursos a los que tengan acceso. Estos factores influenciarán también las formas y mecanismos de poder, que puede ser visible, oculto o invisible. Las primeras dos formas pueden ser mantenidas o resistidas a través de los mecanismos de coerción, la manipulación, la autoridad y la influencia. Por otra parte, el poder invisible depende de patrones sociales y culturales. En suma, los recursos, la agencia y el poder de las personas determinan los logros de una persona, o lo que esta ha logrado ser o hacer.

Por otra parte, las normas sociales influyen en las formas, mecanismos de poder, los recursos disponibles a cada miembro del hogar y la agencia de los individuos. Al ser tan omnímodo, es imprescindible ahondar en la relación entre las normas sociales y el poder. Este es el propósito de las siguientes secciones. En primer lugar, se ahondará en el significado de las normas sociales, y en segundo, basado en la literatura existente, se analizará la influencia de las normas sociales en el poder de los miembros del hogar y por ende, en su bienestar. 
Figura 2: El poder relacional en el hogar

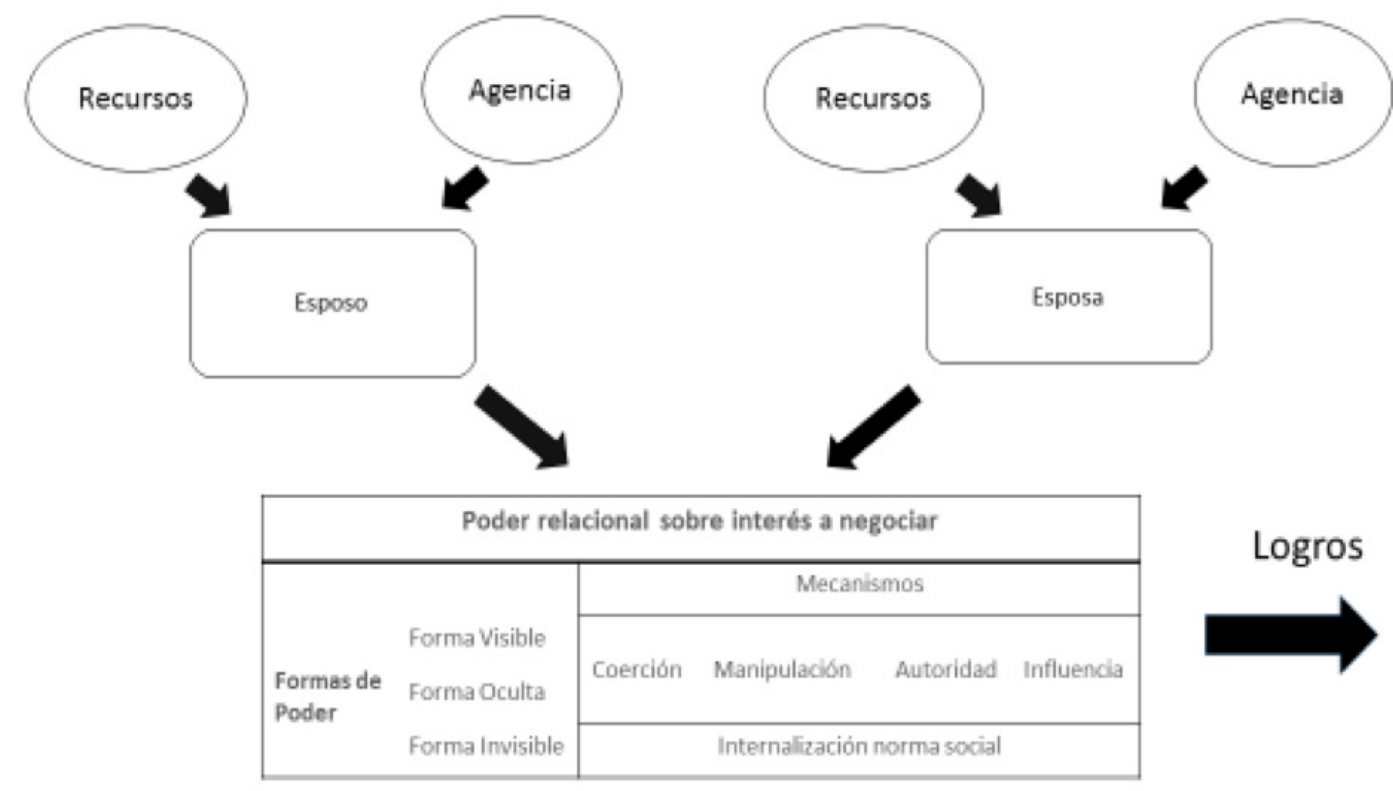

Fuente: Elaboración propia.

\section{2. ¿Qué son las normas sociales?}

Las normas sociales son los factores más reconocidos en la literatura como determinantes del empoderamiento de las mujeres. ¿Pero qué son las normas sociales? No existe un consenso entre los académicos sobre una definición de normas sociales. Generalmente el significado depende del enfoque del autor. Incluso, existen una serie de términos, tales como las costumbres, las convenciones, las instituciones, roles, la cultura, etc., que son similares e intersectan con la noción de normas sociales (Horne, 2001; Bicchieri, 2005). La definición usada aquí, es una modificación de la propuesta por Rutherford (1996) y usada en Covarrubias (2016). Que considera las normas sociales como reglas informales de moralidad (reglas que conciernen los principios de comportamiento correcto e incorrecto) que son sostenidas por un grupo de individuos de la sociedad. La característica que distingue a las normas sociales de otras reglas sociales es justamente que son de tipo "moral". Una convención, es también una regla sostenida por un grupo de individuos en la sociedad, donde todos conforman con la regla ya que es la acción que conviene realizar al grupo. Por ejemplo, en México se conduce por el lado derecho, y si alguien manejara del lado izquierdo, chocaría (Young, 1996). Las normas también se diferencian de los hábitos y las rutinas, que son reglas de moralidad personal y de consciencia privada, mantenidas por inercia y no tienen mayor significado social más que el personal (Rutherford, 1996). Por último, las reglas legales a diferencia de las normas sociales, porque son ejecutadas por un sistema judicial y policiaco, que castiga las violaciones a las normas (Elster, 1989).

Existen dos mecanismos que hacen que las normas sociales sean cumplidas (al menos por un grupo de personas) en una sociedad; la internalización y las sanciones sociales. En cuanto al primer mecanis- 
mo, debido a que las normas sociales son reglas morales, las personas que se adscriben a ellas, tendrán sentimientos de remordimiento y culpa si no las cumplen. El proceso de socialización instala en la conciencia o en el súper ego una especie de sistema interno de vigilancia (Coleman, 1990). A este fenómeno se le conoce como internalización de las normas sociales.

Las normas varían en su nivel de subjetividad, esto es, la medida en la cual una norma ha sido naturalizada o internalizada por personas en la sociedad. "Cada orden establecido tiende a producir (a diferentes niveles y a través de distintos medios) la naturalización de su propia arbitrariedad. De todos los mecanismos que tienden a producir este efecto, el más importante y el más encubierto, es sin duda la dialéctica de las oportunidades objetivas y de las aspiraciones de los individuos, de la cual surge el sentido de límites, comúnmente llamado el sentido de realidad. Cuando, debido al ajuste casi perfecto entre las estructuras objetivas y las estructuras internalizadas que resulta de la lógica de la simple reproducción, el orden político y cosmológico establecido es percibido no como arbitrario i.e. como uno posible entre otros, sino como evidente y como un orden natural que va sin ser cuestionado y por lo tanto continua sin ser cuestionado. Las aspiraciones de los agentes tienen los límites como las condiciones objetivas de las cuales son productos (Bourdieu, 1977: 164)." Bourdieu (1977) le llama a la experiencia doxa para distinguirla de otras creencias que implican conciencia y reconocimiento de la posibilidad de creencias antagonistas. El nivel de subjetividad determinara la medida en la cual una norma es extendida en la comunidad.

¿Pero cómo puede una norma dóxica ser revelada en la sociedad? "La verdad de doxa solo es revelada cuando es negativamente constituida por el campo de la opinión, en el centro neurálgico de la confrontación de discursos conflictivos- cuya verdad política solo puede ser declarada abiertamente o permanecer escondida, sobre todo de los ojos que se involucraron con ella, debajo de oposiciones disfrazadas de religiosas y filosóficas" (Bourdieu, 1977: 168).

En suma, habrá normas sociales que estarán menos interiorizadas por la población, y por lo tanto una menor cantidad de personas la seguirán. Por ejemplo, hay grupos cristianos que tienen como norma no recibir una transfusión de sangre, es una norma moral, en la cual cree y sigue un grupo minoritario de la sociedad. Hay otras normas, que están tan interiorizadas y naturalizadas, que no son cuestionadas, son 'doxicas'. En muchas sociedades, por ejemplo, el que los hombres sean las amas de casa es impensable, el orden natural es que las mujeres realicen esa labor.

Debido a que las normas sociales son compartidas por un grupo de personas que coinciden en su visión de lo que es propio, si una persona del grupo rompe la regla, las otras se sentirán con el derecho de expresar su desaprobación ya sea criticando a la persona o difundiendo chismes sobre ella. A veces las normas y los argumentos que las validan son compartidas por un gran segmento de la sociedad y en otros casos serán apoyadas solo por un pequeño grupo (Elster 1989). Para que los chismes ocurran, las personas que están involucradas tienen que ser parte del segmento de la sociedad que aprueba la norma, esto es, tienen la misma idea de lo que es propio. Las sanciones sociales pueden tomar la forma de chismes, críticas, y hasta violencia y ostracismo.

De acuerdo a Merry (1984), los chismes crean mapas cognitivos de identidades sociales y reputaciones. Crea dossiers de cada miembro de una comunidad: quién es un buen curador, quien es un ladrón, quién es una bruja y quién es un trabajador. A diferentes grados, a la gente le interesa su imagen en una sociedad y por lo tanto experimentará angustia cuando se generan chismes sobre ella. 


\section{Normas sociales y poder de las mujeres en el hogar}

El rol socialmente asignado a las mujeres es el de ser amas de casa, de cuidar de los niños, enfermos y ancianos, de ser fieles y sexualmente pudorosas, de obedecer a sus esposos, de permanecer en el hogar y cuidar de él. Por otra parte, se espera que los esposos sean los proveedores económicos, la autoridad en el hogar, que sean sexualmente activos, que tengan fuerza física, que tengan control sobre el cuerpo de las mujeres, y que controlen y manejen los recursos económicos. Estas normas sociales, tendrán importantes efectos en el poder relacional de pareja. Con base en resultados de estudios empíricos, a continuación, se describen algunos de los canales a través de los cuales las normas sociales influyen en el poder y empoderamiento de las mujeres en el hogar.

En México, existe una estructura jerárquica en el hogar, en el que los hombres tienen un mayor poder sobre otros miembros del hogar. Esta jerarquía en parte es sostenida por la autoridad que se le confiere a los esposos y jefes de hogar en varias comunidades. En un estudio sobre la participación de las mujeres en las maquilas de Tehuacán, Covarrubias (2010) encontró que por lo menos en lo discursivo, las esposas tenían que pedir permiso a sus esposos para trabajar. Appendini (2009) también encontró este patrón en mujeres de mediana edad en tres comunidades de estados que bordean la Ciudad de México (el Estado de México, Morelos y Querétaro). En ambos estudios, las esposas pedían permiso y obedecían la decisión de los varones a pesar de que generalmente ellos se negaban a que las mujeres trabajaran. En otros países, se percibe incluso a las esposas como si fueran una propiedad. Por ejemplo, Silberschmidt (1992) encontró en el distrito Kisii en Kenya, que las mujeres indicaban 'nos compran como si fuéramos ganado'. Los esposos tenían que ser consultados en todo tipo de cuestiones y determinaban todas las acciones que tenían que tomar. El hecho de que las mujeres tengan que pedir permiso a los varones, o consultarlos para que ellos tomen las decisiones, teniendo ellos la autoridad de decidir sobre aspectos fundamentales sobre sus vidas, implica que no son consideradas adultas en su propio juicio, su sentido de agencia está siendo subvalorado. Vemos entonces, que las normas sociales influyen en el poder al otorgar la autoridad a los esposos de tomar decisiones sobre sus parejas.

Dada la autoridad de los esposos, el que sus parejas se involucren en un proceso de negociación o no, es decir, que la forma de poder sea visible u oculto, dependerá tanto de los resultados esperados de esa negociación y de su propia agencia. Si la esposa sabe que su esposo estará en desacuerdo y no le dará permiso bajo ninguna circunstancia para hacer lo que a ella le interesa, entonces no tendrá ningún incentivo para negociar. Incluso, habrá un desincentivo, si espera que la autoridad del hombre sea reforzado con algún tipo de coerción, como por ejemplo la violencia física o verbal como se verá más adelante. Por otra parte, si la mujer no tiene agencia, es decir le cuesta trabajo decidir y actuar en consecuencia, entonces la forma de poder será oculta. Esta también es influenciada por las normas sociales como se verá más adelante. Covarrubias (2010), encontró, por ejemplo, que de las mujeres que no trabajaban en la maquila, el 33\% querían trabajar, pero sus esposos no les daban permiso, y el 9\%, quería trabajar y nunca le había dicho a su esposo que quería hacerlo.

La violencia es también una manera de coerción que es utilizada para mantener el poder en el hogar y ésta, es justificada por las normas sociales de género. De acuerdo con un estudio que realizó Contre- 
ras Urbina (2008) en la Ciudad de México, las normas sociales dan la legitimidad y autorización a los hombres de disciplinar a su mujer si esta no cumple con sus roles de género. Esto también se da en otras partes del mundo. En Zimbabue, por ejemplo, más de la mitad de las mujeres de entre 15-49 años creen que está justificado que a las mujeres les peguen ya sea porque se rehúsan a tener sexo con sus esposos, debido a que sale sin su permiso, si discute con él o si él percibe que no cuida bien a los hijos o no hizo bien la comida (Hindin, 2003).

También se ha encontrado evidencia para la teoría de la inconsistencia de estatus, usada en la literatura de violencia familiar. Esta afirma que los varones que sienten amenazados ya sea por no poder cumplir su rol de proveedores económicos o por la pérdida de estatus o autoridad en la familia, tienen una mayor incidencia de emplear violencia (Casique, 2008). Es decir, se ha encontrado que la violencia es usada por los hombres para reforzar su autoridad cuando sienten que la pierden. Contreras (2008) encontró evidencia de esta teoría en la Ciudad de México, realizando entrevistas a hombres de estatus socioeconómico "medio-bajo" que trabajaban en fábricas. Para ellos la violencia también estaba socialmente legitimada, en el caso de que los esposos tuvieran que defender su autoridad en caso de una supuesta agresión, física o verbal, y así no ser identificados como mandilones. Es decir, el mecanismo de la violencia, un mecanismo grave de ejercer poder, podía ser usado, en caso de que las mujeres no respetaran la autoridad socialmente aceptada de los hombres. Esta forma de poder de los varones sobre las esposas es visible, ya que el conflicto es manifestado. Sin embargo, dada la experiencia, y el conocimiento de la posibilidad de violencia de los esposos, sus parejas pueden en un futuro de negociar por sus intereses y aceptar la autoridad y decisiones de los varones, reduciendo la probabilidad de que el poder sea visible y en este caso sea oculto.

A pesar de que existe una estructura jerárquica en el hogar a favor de los esposos, se han encontrado ejemplos de resistencia oculta por parte de las mujeres que involucran la manipulación. Regresando al ejemplo de Silberschmidt (1992) en Kenya, dado que la tierra pertenece al hombre, se espera que él decida sobre qué cultivar. Si la esposa estuviera en desacuerdo con esta práctica, rara vez diría algo, pero simplemente sembraría como ella pensara que es la mejor forma. Si él se entera que no siguió sus instrucciones, ella se disculparía pero explicaría que debido a que la semilla no había germinado, había tenido que ser replantado de otra forma. Esto es a lo que Kabeer (1999) llama forma privada e informal de empoderamiento, ya que deja intacto la autoridad, la imagen, y el honor público tradicional de los esposos, pero incrementa la influencia de las mujeres en el proceso de negociación. Por lo tanto, ante el poder que las normas sociales otorgan a los varones, las esposas tienen una forma de poder oculto, pero utilizando los mecanismos de la manipulación y el engaño, es decir recurren a encubrir sus acciones para poder tomar decisiones.

Se ha encontrado que las normas sociales, al legitimar ciertas acciones, también afectan el poder visible de las personas, al ser un argumento para la persuasión. Por ejemplo, utilizando información de entrevistas a profundidad a mujeres en la frontera mexicana, Gates (2002) encontró que para negociar su incorporación al trabajo asalariado, las mujeres casadas a veces ofrecían hacer más labores dentro del hogar, como una estrategia para ganarse el derecho a trabajar. Otras también ofrecían contribuir económicamente a comprar cosas grandes como una casa (Covarrubias, 2010).

Las normas sociales de género, también influyen en que las esposas estén desempoderadas de forma invisible. En una sociedad en donde una norma social es dóxica, es decir, está naturalizada, nadie va a 
cuestionarla, por lo que se convertirá en una regla moral que es seguida sin cuestionamientos. Por lo tanto, cualquier acción o decisión contraria a las normas sociales de género, como el trabajo de las mujeres fuera del hogar, el control de recursos de las mujeres, el que las mujeres puedan negarse a tener relaciones sexuales, no será siquiera cuestionado.

Por otra parte, Sen (1987a) explica que las normas sociales de género, al prescribir distintos comportamientos y cualidades entre hombres y mujeres, influyen en la percepción de sus preferencias, o lo que él llama "intereses percibidos". Por ejemplo, hay muchas mujeres que cuando se le pregunta sobre su bienestar, hablan en términos del bienestar de su familia. En este sentido, Brickell y Chant (2010) elaboran cómo en varias culturas, a las mujeres se les condiciona a ser madres y esposas altruistas, mientras que a los padres y esposos no. Debido a los atributos, supuestamente naturales, adscritos a las mujeres, de cuidadoras de hijos, enfermos y ancianos, éstas priorizan las necesidades de otros, especialmente los de sus familias, antes que a las propias. Mientras tanto a los hombres se les califica como egoístas e inestables. Un ejemplo de ello es la doble carga de trabajo que realizan las mujeres que tienen una actividad económica, ya que además de dedicarse a esta labor, llegan a su casa a hacer si no todas, la mayor parte de las labores domésticas y el cuidado de sus hijos. Esta doble carga es explicada en la sociedad, (incluso por las propias mujeres), con los argumentos de que la naturaleza las dotó de cualidades para ser cuidadoras (limpiadoras y cocineras) y a la ausencia de opciones alternativas, pero no a la irresponsabilidad paterna. También se tiene evidencia de que, en algunas regiones y países, como en Tanzania, India y Bangladesh, las mujeres son las últimas de su familia en comer alimentos y ellas dan a sus esposos e hijos varones la mejor comida del hogar. Por lo tanto, mientras que a las mujeres se les condiciona socialmente a poner las necesidades y preferencias de sus esposos e hijos antes que los propios, por lo que sus 'intereses percibidos' no estarán en función de su bienestar.

Las normas sociales también influyen en el poder de los miembros del hogar al afectar la agencia de los individuos. En este sentido, muchas mujeres internalizan su estatus social como personas de menor valor (Kabeer 1999). Nussbaum (2000) explica como muchas mujeres no identifican el abuso y la violación de sus derechos humanos y jurídicos, como tales. No se reconocen como titulares de derechos y como ciudadanas cuyo sentido de valor y dignidad es igual al de otros. Es decir, las normas sociales perjudican su autoestima y sentido de valor. Además, las normas sociales validan la conducta que deben de seguir los hombres y las mujeres. Por ejemplo, en varias culturas las mujeres tienen que ser calladas y sumisas, mientras que los varones son fuertes (Nussbaum, 2000). De esta forma, las mujeres no se sentirán libres de expresar sus deseos e intereses, por lo que no negociarán abiertamente ni explícitamente con sus esposos o utilizarán formas de resistencia ocultas.

Por último, las normas sociales influyen en el poder de los individuos indirectamente, al influir en el acceso y control de los recursos sociales, políticos y económicos. Por ejemplo, en México, las mujeres se encuentran en una posición desfavorable en el proceso de herencia de tierra, en las zonas rurales, debido que los padres prefieren dar sus tierras a los hijos varones, ya que son ellos los que tienen el rol social como proveedores económicos del hogar. Pocas mujeres están conscientes de su derecho a heredar, aunque la herencia representa para ellas el medio más importante para obtener tierra (Ramírez, 2011).

También hay muchas menos mujeres con trabajos fuera de su hogar. Las normas sociales también influyen directamente en la participación laboral de las mujeres. Varios estudios econométricos rela- 
cionan las normas sociales y el trabajo asalariado de las mujeres. Covarrubias (2016) encuentra que, en Tehuacán, la internalización de tres reglas morales influye en la participación laboral de las mujeres. La primera es que las esposas son las responsables de las tareas domésticas y del cuidado de niños, enfermos y ancianos. La segunda indica que las mujeres que trabajan en la maquila son promiscuas. La tercera que los esposos tienen que ser los proveedores económicos en el hogar. Fortin (2005) en un estudio econométrico de 25 países de la OECD, relaciona la participación laboral de las mujeres con la percepción de los individuos sobre roles sociales tales como, "Cuando los empleos son escasos los hombres tienen más derechos a un empleo que una mujer" y "Ser una ama de casa es tan gratificante como trabajar por un ingreso", y encuentra que esta relación es significativa.

Los modelos económicos neoclásicos de negociación dentro del hogar, indican que el ingreso y los activos en posesión de los miembros del hogar influyen en la posibilidad de dejar un matrimonio o relación (Katz, 1997). Por ejemplo, una mujer que tiene un empleo y gana lo suficiente, tiene mayor posibilidad de abandonar su matrimonio al poder mantenerse a ella misma y a sus hijos, por lo que podrá exigir una mayor participación en las decisiones importantes dentro del hogar. Ariza y Oliveira (2002) indican que la generación de ingresos también permite a las mujeres redefinir las fronteras de autoridad, obediencia y violencia masculina.

Otro motivo por el cual la generación de ingresos conlleva un mayor poder dentro del hogar es elaborado por Sen (1987a), quien introduce la noción de contribución percibida, es decir la percepción de que se está haciendo una mayor contribución al hogar, legitimando así la adjudicación de una mayor proporción de los ingresos y participación en las decisiones. El que las mujeres no generen un ingreso no significa que no aporten al hogar. El llevar a cabo las labores domésticas y de cuidado de los hijos requiere de un esfuerzo y trabajo sustancial, no obstante, este trabajo no es reconocido y legitimado de la misma forma que la generación de ingresos. La contribución percibida al hogar depende más del aporte económico que al esfuerzo y tiempo dedicado a las actividades.

No obstante, el ingreso que las mujeres obtienen por participar en actividades económicas no siempre traduce per se en un mayor poder para ellas. Kantor (2003) por ejemplo, analiza econométricamente si mujeres en India, que son auto-empleadas y miembros del grupo SEWA (Self Employed Women's Association), toman mayores decisiones importantes dentro del hogar como la venta de propiedades, el matrimonio de algún miembro de la familia, su movilidad, etc., dependiendo del ingreso que obtienen. Encontró que un mayor ingreso proveniente del autoempleo, incluso si este representaba más del 50\% de los ingresos del hogar, no se traducía en una mayor probabilidad de participación para las mujeres en las decisiones del hogar, a menos que tuviera control sobre esos ingresos. Esto implica que la relación entre ingresos y empoderamiento no es tan directa como se cree, si no que presenta un carácter más complejo. Es posible que existan normas sociales que conlleven a que esta relación sea más enredada.

Como puede deducirse, los canales a través de los cuales las normas sociales influyen en el poder de las mujeres en el hogar, son numerosos. En este artículo se mencionan varios, pero es necesario continuar indagando en esta cuestión, para así tener una idea más clara de los mecanismos para su empoderamiento $\mathrm{y}$, por lo tanto, contar con políticas públicas exitosas, encaminadas a este propósito. 


\section{Conclusión}

Se utilizó un marco teórico y conceptual para analizar el poder relacional en el hogar. Es natural que el conflicto de intereses se dé en este ámbito. Esto dependerá de la visibilidad y mecanismos que se utilicen para mantener y ejercer el poder. Generalmente, debido al modelo jerárquico de muchas comunidades, las mujeres son las que tienen un menor poder de incidir sobre sus intereses y decisiones en el hogar. En este artículo se mostró que hay evidencia que sugiere que los varones tienen autoridad sobre las mujeres en varias decisiones estratégicas en la vida de ellas, tales como su libertad de movimiento, y su trabajo. Esto implica que no son consideradas sujetos con agencia y adultos con poder de decidir sobre aspectos fundamentales en su vida. Incluso hay estudios que señalan que los varones pueden llegar a usar la violencia ya sea verbal o física, si sus parejas desafían su autoridad. Es evidente que esto repercute negativamente en el bienestar de las mujeres. Por el otro lado, las mujeres utilizan mecanismos ocultos, tales como el engaño para resistir el poder que sus esposos tienen sobre ellas. Por lo tanto, todas las formas de resolución de estos conflictos, tendrán el mismo efecto en el bienestar de los miembros. Para empoderar a las mujeres, es importante ahondar en estas complejidades. El marco presentado, tiene la ventaja de reconocer que en el hogar surgen conflictos de intereses y evidenciar la visibilidad y mecanismos por los cuales los individuos mantienen poder. El marco teórico de empoderamiento comúnmente utilizado por feministas no considera estas cuestiones.

Para que todos los miembros del hogar obtengan bienestar de sus relaciones, es necesario que tengan el acceso a negociar y debatir sus intereses abierta y visiblemente, sin miedo a represalias internas, de la pareja o por parte de la sociedad. Una propuesta que cumple con estos requisitos es la elaborada por Schmukler (2013: 204) que "promueve procesos de corresponsabilidad entre los géneros, reconocimiento de la autoridad de las mujeres equitativamente con los hombres, reconocimiento de los niños y niñas como sujetos de derechos y agentes activos en la toma de decisiones familiares, transformar el adultismo en un vínculo de escucha emocional y respeto de madres, padres e hijos."

Para proponer proyectos y políticas públicas que lleven a este ideal, es fundamental conocer los factores que influyan en el empoderamiento de las mujeres en este ámbito, es decir la forma en que los recursos incluyendo las normas sociales y la agencia influyen en el poder de los individuos. Para esto es importante que se realice trabajo de campo usando el marco propuesto en este artículo.

El presente marco, sirvió para analizar a profundidad las normas sociales, uno de los factores que ha sido identificado ampliamente en la literatura como determinante del empoderamiento. Como se ha visto, debido a la gran extensión e internalización de las normas sociales, los conductos a través de los cuales éstos influyen en el poder de las mujeres son múltiples, por lo que es importante identificar y estudiar empíricamente cada uno. Es fundamental reconocer también que las normas sociales no solo influyen directamente en la agencia de los individuos, también afectan el acceso y beneficio obtenido de los recursos económicos, sociales y políticos, a través de los cuales se pueden empoderar.

Éste artículo se hizo para examinar el poder a nivel relacional. Además de la importancia de ir construyendo sobre él, es necesario que también se adecúe el presente marco para que el poder de las mujeres a nivel comunitario también pueda ser analizado. Es a nivel comunitario donde las mujeres pueden obtener también recursos e infraestructura, abogar por sus derechos, y apoyarse mutuamente. 


\section{Referencias}

Agarwal, Bina. 1997. Bargaining and Gender Relations: within and beyond the Household. Feminist Economics 3(1): 1-51. DOI: https://doi.org/10.1080/135457097338799

Ariza, Marina y Orlandina Oliveira. 2002. Cambios y continuidades en el trabajo, la familia y la condición de las mujeres. En Estudios Sobre las Mujeres y las Relaciones de Género en México: Aportes Desde Diversas Disciplinas compilado por Elena Urrutia, pp. 83-87. Ciudad de México: El Colegio de México. Appendini, Kirsten. 2009. Economic liberalization, changing livelihoods and gender dimensions in rural Mexico. Reporte presentado en el taller Gender dimensions of agricultural and rural employment: differentiated pathways out of poverty Part III. Roma: FAO, IFAD and ILO. http://www.fao-ilo.org/fileadmin/ user_upload/fao_ilo/pdf/Papers/16_march/Appendini_-fin2.pdf

Bicchieri, Cristina. 2005. The Grammar of society: The nature and dynamics of social norms. Londres: Cambridge University Press.

Bourdieu, Pierre. 1977. Outline of a theory of practice. Londres: Cambridge University Press.

Brickell, Katherine y Sylvia Chant. 2010. The unbearable heaviness of being' reflections on female altruism in Cambodia, Philippines, The Gambia and Costa Rica. Progress in Development Studies 10(2): 145159. DOI: https://doi.org/10.1177/146499340901000204

Casique, Irene. 2008. El Complejo vínculo entre el empoderamiento de la mujer y violencia de género. En Estudios sobre cultura, género y violencia contra las mujeres compilado por Roberto Castro e Irene Casique, pp. 231-260. Ciudad de México: UNAM.

Coleman, James. 1990. Foundations of social theory. Cambridge y Londres: Belknap Press of Harvard University Press.

Contreras, Juan. 2008. La legitimidad social de la violencia contra las mujeres en la pareja. Un estudio cualitativo de varones en la Ciudad de México. En Estudios sobre cultura, género y violencia contra las mujeres compilado por Roberto Castro e Irene Casique, pp. 41-80. Ciudad de México: UNAM.

Covarrubias, Arlette. 2010. Women's deviation from gendered social norms: Assembly plant employment in Tehuacan, Mexico. Tesis doctorado en desarrollo internacional, Universidad de East Anglia.

Covarrubias, Arlette. 2016. La sombre voluntaria. Normas sociales y participación laboral de las mujeres en la maquila. Estado de México: El Colegio Mexiquense, A.C.

Elster, Jon. 1989. The Cement of society: The study of social order. Cambridge: Cambridge University Press. Fortin, Nicole. 2005. Gender role attitudes and the labour-market outcomes of women across OECD countries. Oxford Review of Economic Policy 21(3): 416-438. DOI: https://doi.org/10.1093/oxrep/gri024 Gaventa, Jonathan. 2011. Power pack, understanding power for social change. http://www.powercube. net/wp-content/uploads/2011/04/powerpack-web-version-2011.pdf (15 abril 2016).

Gates, Leslie. 2002. The strategic uses of gender in household negotiations: Women workers on Mexico's northern border. Bulletin of Latin American Research 21(4): 507-526. DOI: https://doi.org/10.1111/14709856.00057

Hindin, Michelle. 2003. Understanding women's attitudes towards wife beating in Zimbabwe. Bulletin of the World Health Organization 81(7): 501-508.

Horne, Christine. 2001. The enforcement of norms: Group cohesion and meta-norms. Social Psychology 
Quarterly 64(3): 253-266. DOI: https://doi.org/10.2307/3090115

Iversen, Vegard. 2003. Intra-household inequality: A challenge for the capability approach. Feminist Economics 9(2-3): 93-115. DOI: https://doi.org/10.1080/1354570032000080868

Kabeer, Naila. 1999. Resources, agency, achievements: Reflections on the measurement of women's empowerment. Development and Change, 30(3): 435-464. DOI: https://doi.org/10.1111/1467-7660.00125

Kantor, Paula. 2003. Women's empowerment through home-based work: Evidence from India. Development and Change, 34(3): 425-445. DOI: https://doi.org/10.1111/1467-7660.00313

Katz, Elizabeth. 1997. The intra-household economics of voice and exit. Feminist Economics, 3(3): 25-46. DOI: https://doi.org/10.1080/135457097338645

Lukes, Steven. 2005. Power: A radical view. Basingstoke: Palgrave Macmillan.

Malhotra, Anju, Sidney Schuler y Carol Boender. 2005. Measuring women's empowerment as a variable in international development. Background Paper. Washington: World Bank. http://siteresources.worldbank.org/INTGENDER/Resources/MalhotraSchulerBoender.pdf

Merry, Sally. 1984. Rethinking gossip and scandal. En Toward a general theory of social control compilado por Donald Black, pp. 271-302. Nueva York y Londres: Academic Press.

Nussbaum, Martha. 2000. Women and human development: The capabilities approach. Cambridge: Cambridge University Press.

Ramírez, Diana. 2011. Productividad agrícola de la mujer rural en Centroamérica y México. Documento de trabajo. Ciudad de México: CEPAL. http://repositorio.cepal.org/bitstream/handle/11362/26078/ S2011148_es.pdf;jsessionid=9B365847BEF388303750C40151BCB332? sequence $=1$

Rowlands, Jo. 1997. Questioning empowerment: Working with women in Honduras. Oxford: Oxfam.

Rutherford, Malcolm. 1996. Institutions in economics: The old and the new institutionalism. Cambridge: Cambridge University Press.

Schmukler, Beatriz. 2013. Democratización familiar como enfoque de prevención de violencia de género: experiencias en México. Revista latinoamericana de estudios de familia 5: 199-221.

Scott, John. 2001. Power (key concepts). Cambridge: Polity Press.

Sen, Amartya.1985a. Commodities and capabilities. Amsterdam: North Holland.

Sen, Amartya. 1985b.Well-being, agency and freedom: the Dewey lectures 1984. The Journal of Philosophy, 82(4), pp.169-221. DOI: https://doi.org/10.2307/2026184

Sen, Amartya. 1987a. Gender and cooperative conflicts. WIDER Working Papers 18. Helsinki: World Institute for Development Economics Research. United Nations University. https://www.wider.unu.edu/ sites/default/files/WP18.pdf

Sen, Amartya. 1987b.The standard of living: lecture I, concepts and critiques. En The Standard of Living compilado por Goeffrey Hawthorn, pp 1-19. Cambridge: Cambridge University Press.

Sen, Amartya. 1990. Development as capability expansion. En Human development and the international development strategy for the 1990s compilado por Keith B. Griffin y John B. Knight, pp 41-58. Houndmills: Macmillan/ United Nations.

Sen, Amartya.1992. Inequality re-examined. Oxford: Clarendon Press.

Sen, Amartya. 1993. Capability and well-being, En The quality of life compilado por Martha Nussbaum y Amartya Sen, pp 30-53. Oxford: Clarendon Press.

Sen, Amartya. 1999. Development as freedom. Nueva York: Knopf. 
- Revista de Ciencias Sociales y Humanidades. ISSN-P: 0188-9834 ISSN-E:2395-8669.

Silberschmidt, Margrethe. 1992. Have men become the weaker sex? Changing life situations in Kisii District, Kenya. The Journal of Modern African Studies 2 (30): 237-253. DOI: https://doi.org/10.1017/ S0022278X00010703

Spinoza, Benedictus. 2002. Tractatus politicus. En Spinoza: The Complete works compilado por Michael L. Morgan, pp 676-754. Indianapolis/Cambridge: Hackett Publishing.

Young, Peyton. 1996. The Economics of convention. Journal of Economic Perspectives, 10(2): 105-122. DOI: https://doi.org/10.1257/jep.10.2.105 